\title{
ITERATIVE METHOD FOR SOLVING A BOUNDARY VALUE PROBLEM FOR BIHARMONIC TYPE EQUATION*
}

\author{
DANG QUANG A ${ }^{1}$, LE TUNG SON ${ }^{2}$ \\ ${ }^{1}$ Institute of Information Technology \\ ${ }^{2}$ Thai Nguyen Pedagogic College
}

\begin{abstract}
The solution of boundary value problems (BVP) for fourth order differential equations by their reduction to BVP for second order equations with the aim to use the achievements for the latter ones attracts attention from many researchers. In this paper, using the technique developed by ourselves in recent works, we construct iterative method for a BVP for biharmonic type equation. The performed numerical experiments show very fast convergence of the proposed algorithm.

Tóm tắt. Việc giải các bài toán biên đối với phương trình đạo hàm riêng cấp bốn bằng cách đưa chúng về các bài toán biên đối với phương trình cấp hai đã thu hút sự quan tâm của nhiều tác giá. Trong bài báo này, sử dụng kỹ thuật do chúng tôi phát triển trong nhièu công trình mới đây, một phương pháp lặp giải một bài toán biên cho phương trình song kiểu điều hòa đã được đề xuất. Sự hội tụ nhanh của phương pháp đã được chứng tỏ trên nhiều thực nghiệm tính toán.
\end{abstract}

\section{INTRODUCTION}

The solution of fourth order differential equations by their reduction to boundary value problems (BVP) for the second order equations, with the aim of using efficient algorithms for these, attracts attention from many researchers. Namely, for the biharmonic equation with the Dirichlet boundary condition, there is intensively developed the iterative method, which leads the problem to two problems for the Poisson equation at each iteration (see e.g. [4, 9, 11, 12]). Recently, Abramov and Ulijanova [1] proposed an iterative method for the Dirichlet problem for the biharmonic type equation, but the convergence of the method is not proved. In our previous works $[6,7]$ with the help of boundary or mixed boundary-domain operators appropriately introduced, we constructed iterative methods for biharmonic and biharmonic type equations associated with the Dirichlet boundary condition. Recently, in [8] for solving the Neumann BVP we introduced a purely domain operator for studying the convergence of an iterative method. It is proved that the methods are convergent with the rate of geometric progression. In this paper we develop our technique in [4] - [8] for the following problem

$$
\begin{array}{r}
L u \equiv \Delta^{2} u-a \Delta u+b u=f \text { in } \Omega, \\
u=g_{0}, \quad \frac{\partial \Delta u}{\partial \nu}=g_{1} \text { on } \Gamma,
\end{array}
$$

where $\Delta$ is the Laplace operator, $\Omega$ is a bounded domain in $R^{n}(n \geq 2), \Gamma$ is the sufficiently

\footnotetext{
${ }^{*}$ This work is supported in part by the National Basic Research Program in Natural Sciences, Vietnam
} 
smooth boundary of $\Omega, \nu$ is the outward normal to $\Gamma$ and $a, b$ are positive constants. The equation (1) with other boundary conditions are met, for example, in [2,3,8]. An iterative method reducing the problem to a sequence of Neumann and Dirichlet problems for second order equations will be proposed and investigated by experimental way.

\section{CONSTRUCTION OF ITERATIVE METHOD}

Set $\Delta u=v$ and

$$
\varphi=-b u
$$

Then the problem (1)-(2) is decomposed into the two problems

$$
\begin{gathered}
\Delta v-a v=f+\varphi \text { in } \Omega, \quad \frac{\partial v}{\partial \nu}=g_{1} \text { on } \Gamma . \\
\Delta u=v \text { in } \Omega, \quad u=g_{0} \text { on } \Gamma .
\end{gathered}
$$

From here we see that the solution $u$ of the above problems depends on the function $\varphi$, i.e., $u=u(\varphi)$. Since $u$ and $\varphi$ are related with each other by the relattion (3) we get the functional equation

$$
\varphi+b u(\varphi)=0 .
$$

In order to construct iterative method for finding $\varphi$ first we have to establish the concrete form of the above equation. For the purpose we introduce an operator $B$ defined in the space $L^{2}(\Omega)$ by the formula

$$
B \varphi=b u,
$$

where $u$ is found from the problems

$$
\begin{aligned}
\Delta v-a v & =\varphi \text { in } \Omega, \quad \frac{\partial v}{\partial \nu}=0 \text { on } \Gamma, \\
\Delta u=v & \text { in } \Omega, \quad u=0 \text { on } \Gamma .
\end{aligned}
$$

Proposition 2.1. The problem (4), (5) is reduced to the operator equation

$$
(I+B) \varphi=F,
$$

where

$$
F=-b u_{2}
$$

$u_{2}$ being found from the problem

$$
\begin{aligned}
& \Delta v_{2}-a v_{2}=f \text { in } \Omega, \quad \frac{\partial v_{2}}{\partial \nu}=g_{1} \text { on } \Gamma, \\
& \Delta u_{2}=v_{2} \text { in } \Omega, \quad u_{2}=g_{0} \text { on } \Gamma .
\end{aligned}
$$

Proof. First we notice that the functions $u_{2}$ and $v_{2}$ are completely determined by the data functions of the original problem (1), (2), namely, by $f, g_{0}$ and $g_{1}$. Let $u$ and $v$ be the solutions of the problems (4), (5). Put

$$
u=u_{1}+u_{2}, \quad v=v_{1}+v_{2}
$$


where $v_{1}$ and $u_{1}$ satisfy the problems

$$
\begin{aligned}
& \Delta v_{1}-a v_{1}=\varphi \text { in } \Omega, \quad \frac{\partial v_{1}}{\partial \nu}=0 \text { on } \Gamma, \\
& \Delta u_{1}=v_{1} \text { in } \Omega, \quad u_{1}=0 \text { on } \Gamma .
\end{aligned}
$$

By the definition of $B$ we have

$$
B \varphi=b u_{1} .
$$

Taking into acount (6) from the above equality we obtain the equation (10) with $F$ given by (11). Thus, the proposition is proved.

Now, consider the following two-layer iterative scheme for solving the operator equation $(10)$

$$
\frac{\varphi^{(k+1)}-\varphi^{(k)}}{\tau}+(I+B) \varphi^{(k)}=F, \quad k=0,1,2, \ldots
$$

where $\tau$ is an iterative parameter.

Proposition 2.2. The iterative scheme (16) can be realized by the following iterative process

1. Given $\varphi^{(0)} \in L_{2}(\Omega)$.

2. Knowing $\varphi^{(k)}(x)$ in $\Omega(k=0,1, \ldots)$ solve consecutively two problems

$$
\begin{aligned}
\Delta v^{(k)}-a v^{(k)} & =f+\varphi^{(k)} \text { in } \Omega, \\
\frac{\partial v^{(k)}}{\partial \nu} & =g_{1} \text { on } \Gamma, \\
\Delta u^{(k)} & =v^{(k)} \text { in } \Omega, \\
u^{(k)} & =g_{0} \text { on } \Gamma .
\end{aligned}
$$

3. Compute the new approximation

$$
\varphi^{(k+1)}=(1-\tau) \varphi^{(k)}-\tau b u^{(k)} .
$$

Proof. It is easy to verify that the solution $u^{(k)}$ and $v^{(k)}$ of the problems (15), (16) can be represented in the form

$$
u^{(k)}=u_{1}^{(k)}+u_{2}, \quad v^{(k)}=v_{1}^{(k)}+v_{2}
$$

where $u_{2}, v_{2}$ are solutions of (12), (13) and $u^{(k)}, v^{(k)}$ satisfy (14), (15) with upper indexes $k$. Therefore, according to the definition of $B$ we have

$$
B \varphi^{(k)}=b u_{1}^{(k)}
$$

and consequently,

$$
b u^{(k)}=B \varphi^{(k)}-F .
$$

In view of this, rewriting (19) in the form

$$
\frac{\varphi^{(k+1)}-\varphi^{(k)}}{\tau}+\varphi^{(k)}+b u^{(k)}=0
$$


we obtain the formula (16). Thus, the proposition is proved.

\section{NUMERICAL RESULTS}

As we see from the iterative process described in the Proposition 2.2 at each iteration we have to solve two BVP for second order equations for $v^{(k)}$ and $u^{(k)}$. For this purpose we can use many available efficient algorithms. Here for definiteness we consider the original problem in unit square and construct difference schemes of second order aproximation. Then for solving the obtained systems of grid equations we use the method of complete reduction [13] with computational complexity of $O\left(M N \log _{2} N\right)$.

We perform some limited experiments in MATLAB for testing the convergence of the iterative process (17)-(19) in dependence on iterative parameter $\tau$ and values of $a$ and $b$. The stopping criterion for the iterative process is $\left\|\varphi^{(k+1)}-\varphi^{(k)}\right\|_{\infty}<\varepsilon=h_{1} h_{2}$, where $h_{1}$ and $h_{2}$ are grid stepsizes. In all examples below we take exact solutions $u$ and the functions $f, g_{0}, g_{1}$ are calculated in accordance with the given exact solutions. The results of experiments for $\tau=1$ are presented in tables, where $K$ is the number of iterations, Error $=\left\|u-u_{a p p}\right\|_{\infty}$, $u_{a p p}$ is the computed approximate solution. The computations are carried out on PC Pentium $4 \mathrm{CPU} 1.80 \mathrm{Ghz}$.

Table 1. $u=0.25 x^{4}+0.25 y^{4}+x^{2}+y^{2}, a=1, b=1.5$

\begin{tabular}{|c|c|c|c|c|}
\hline Grid & $h_{1} * h_{2}$ & $K$ & Error & Time $(\mathrm{secs})$ \\
\hline $32 \times 32$ & $9.76 e-4$ & 4 & $2.80 e-4$ & 0.79 \\
\hline $64 \times 64$ & $2.44 e-4$ & 4 & $8086 e-5$ & 2.04 \\
\hline $128 \times 128$ & $6.10 e-5$ & 5 & $1.38 e-5$ & 9.70 \\
\hline $256 \times 256$ & $1.52 e-5$ & 6 & $4.18 e-6$ & 68.20 \\
\hline
\end{tabular}

Table 2. $u=\sin (\pi x) \sin (\pi y), a=1, b=1$

\begin{tabular}{|c|c|c|c|c|}
\hline Grid & $h_{1} * h_{2}$ & $\mathrm{~K}$ & Error & Time $(\mathrm{secs})$ \\
\hline $32 \times 32$ & $9.76 e-4$ & 2 & 0.0014 & 0.66 \\
\hline $64 \times 64$ & $2.44 e-4$ & 2 & $3.73 e-4$ & 1.62 \\
\hline $128 \times 128$ & $6.10 e-5$ & 2 & $9.30 e-5$ & 6.12 \\
\hline $256 \times 256$ & $1.52 e-5$ & 3 & $8.62 e-7$ & 43.07 \\
\hline
\end{tabular}

Table 3. $u=\left(x^{2}-1\right)^{2} e^{y}+\left(y^{2}-1\right) e^{x}, a=1, b=1$

\begin{tabular}{|c|c|c|c|c|}
\hline Grid & $h_{1} * h_{2}$ & $\mathrm{~K}$ & Error & Time $(\mathrm{secs})$ \\
\hline $32 \times 32$ & $9.76 e-4$ & 3 & $9.50 e-4$ & 0.73 \\
\hline $64 \times 64$ & $2.44 e-4$ & 3 & $2.32 e-4$ & 1.79 \\
\hline $128 \times 128$ & $6.10 e-5$ & 4 & $5.98 e-5$ & 8.81 \\
\hline $256 \times 256$ & $1.52 e-5$ & 4 & $1.51 e-5$ & 55.59 \\
\hline
\end{tabular}

From Tables 1-3 we see that the proposed iterative process (17)-(19) with the chosen $\tau=1$ converges very fast. Some experiments with other values of $\tau$ require more iterations.

For the fixed $\tau=1$ we also perform experiments for number of iterations in dependence on $b$ with fixed $a$ and in dependence on $a$ with fixed $b$. The results of the experiments in the case of the exact solution $u=\left(x^{2}-1\right)^{2} e^{y}+\left(y^{2}-1\right) e^{x}$, grid $128 \times 128$ are given in Tables 4, 5 . 
Table 4. Number of iterations

in dependence on $b$ for fixed $a=1$

\begin{tabular}{|c|c|c|c|c|c|c|c|c|c|}
\hline$b$ & 1 & 3 & 5 & 7 & 9 & 11 & 13 & 15 & 16 \\
\hline$K$ & 4 & 7 & 10 & 14 & 20 & 29 & 46 & 95 & 177 \\
\hline
\end{tabular}

Table 5. Number of iterations

in dependence on $a$ for fixed $b=2$

\begin{tabular}{|c|c|c|c|c|c|c|c|c|c|}
\hline$a$ & 2 & 1 & 0.8 & 0.6 & 0.4 & 0.3 & 0.15 & 0.13 & 0.12 \\
\hline$K$ & 4 & 5 & 6 & 7 & 9 & 12 & 41 & 88 & 236 \\
\hline
\end{tabular}

From Tables 4, 5 we see that the proposed iterative process (17)-(19) shows very good convergence for the case $b / a \leqslant 5$ that is met in real plate bending problems.

\section{CONCLUDING REMARK}

In the paper an iterative method for solving the boundary value problem (1)-(2) for biharmonic type equation was proposed. Its idea is to reduce the problem to a sequence of Neumann and Dirichlet problems for second order equations. The fast convergence of the method was shown on various experiments, where the value of iterative parameter $\tau=1$ seems to be optimal. Theoretical proof of this convergence remains an open problem to be studied.

\section{REFERENCES}

[1] A. A. Abramov and V.I. Ulijanova, On a method for solving biharmonic type equation with singularly small parameter, Journal of Comput. Math. and Math. Physics 32 (1992) 567-575 (Russian).

[2] J.P. Aubin, Approximation of Elliptic Boundary-Value Problems, Wiley-Interscience, 1971.

[3] D. Begis, A. Perronet, The club modulef, Numerical Methods in Applied Mathematics, Ed. G. Marchuc and J. L. Lions, Nauka, Novosibirsk, 1982, 212-236 (Russian).

[4] Dang Quang A, On an iterative method for solving a boundary value problem for fourth order differential equation, Math. Physics and Nonlinear Mechanics 10 (1988) 54-59 (Russian).

[5] Dang Quang A, Approximate method for solving an elliptic problem with discontinuous coefficients, Journal of Comput. and Appl. Math. 51 (1994) 193-203.

[6] Dang Quang A, Boundary operator method for approximate solution of biharmonic type equation, Journal of Math. 22 (1\&2) (1994) 114-120.

[7] Dang Quang A, Mixed boundary-domain operator in approximate solution of biharmonic type equation, Vietnam Journal of Math. 26 (1998) 243-252.

[8] Dang Quang A, Iterative method for solving the Neumann boundary value problem for biharmonic type equation, Journal of Computational and Applied Mathematics 196 (2006) $634-643$. 
[9] A. Dorodnisyn, N. Meller, On some approches to the solution of the stationary NavierStoke equation, Journal of Comp. Math. and Math. Physics. 8 (1968) 393-402 (Russian).

[10] P. Gervasio, Homogeneous and heterogeneous domain decomposition methods for plate bending problems, Comput. Methods Appl. Mech. Engrg. 194 (2005) 4321-4343.

[11] R. Glowinski, J.-L. Lions and R. Tremoliere, Analyse Numerique des Inequations Variationelles, Dunod, Paris, 1976.

[12] B. V. Palsev, On the expansion of the Dirichlet problem and a mixed problem for biharmonic equation into a seriaes of decomposed problems, Journal of Comput. Math. and Math. Physics 6 (1) (1966) 43-51 (Russian).

[13] A. Samarskii and E. Nikolaev, Numerical Methods for Grid Equations, Vol. 2, Birkhäuser, Basel, 1989.

Received on March 17, 2006

Revised on July 30, 2006 